\title{
A Study of Uropathogenic ESBL Producing Gram Negative Bacilli in a Teaching Hospital
}

\author{
Gitanjali Kailas Badave, Qader Ahmed Jalily ${ }^{*}$ and G. Sasikala \\ Department of Microbiology, Mahavir Institute of Medical Sciences, \#2-4-40, Shivareddypet, \\ Vikarabad, Ranga Reddy - 501 101, Telangana, India \\ *Corresponding author
}

\section{Keywords}

Uropathogens, Extendedspectrum beta-lactamase (ESBL), Gram negative,

Community acquired infection, Hospital acquired infection

\section{Article Info}

Accepted: 08 August 2018 Available Online: 10 September 2018

\section{A B S T R A C T}

About 150 million people suffer from urinary tract infection each year. In majority of cases, treatment is initiated empirically based on the antimicrobial resistance pattern of the urinary pathogens prevalent in a particular setting. Emerging antibiotic resistance among Enterobacteriaceae has posed challenges in choosing empiric regimens. Therefore, the present study was designed to identify etiological agents of urinary tract infections, detect ESBL producing uropathogens and study their antibiotic resistance profile. Around 306 urine (Midstream urine and catheterized) samples were collected and processed by semiquantitative culture on Cysteine Lactose Electrolyte Deficient media, blood agar, and MacConkey agar by standard loop method. Bacterial colony count more than $10^{5}$ colonyforming units (CFU)/ml was taken as significant bacteriuria. Antibiotic sensitivity testing was done by Kirby-Bauer disc diffusion method as per Clinical and Laboratory Standards Institute guidelines. ESBL screening and phenotypic confirmation was done by testing the strain against ceftazidime and ceftazidime/clavulanic acid, cefotaxime and cefotaxime/clavulanic acid). Out of 306 urine samples collected, significant bacteriuria was observed in $92.1 \%$ (282/306) samples. Out of the culture positive 282 specimens, female patients reported $72.4 \%$ growth whereas male patients reported $27.6 \%$ growth. Out of 282 isolates, $96.1 \%$ isolates were Gram-negative, E. coli being predominant isolate whereas $3.9 \%$ were Gram-positive isolates (including Candida spp).The isolates showed least resistance to Imipenem (12\%), followed by Nitrofurantoin (24\%), Gentamicin (28\%) Piperacillin Tazobactam (36\%). Higher resistance was reported for Norfloxacin (80\%), Cefazolin (76\%), and Cotrimoxazole (68\%) Ciprofloxcin (64\%), Tetracycline (60\%). Among the Gram negative isolates, 40.2\% (109/271) were found to be ESBL producers.

\section{Introduction}

About 150 million people suffer from urinary tract infection (UTI) each year (Flores-Mireles et al., 2015). Although UTI's occur in all age groups including men and women, clinical studies suggest that the overall prevalence of UTI is higher in women (Salvatore $\mathrm{S}$ et al., 2011) Escherichia coli is the most common cause of (80-85\%) of community-acquired urinary tract infections (Nicolle et al., 2008). Rarely UTI may be due to viral or fungal infections (Amdekar et al., 2011). Healthcareassociated urinary tract infections mainly from urinary catheterization involve a much broader range of pathogens including E. coli, Klebsiella spp, Pseudomonas aeruginosa, Proteus spp, Candida albicans, and 
Enterococcus faecalis etc. (Sievert et al., 12013; Bagshaw et al., 2006) In majority of cases, treatment is initiated empirically based on the antimicrobial resistance pattern of the urinary pathogens prevalent in a particular setting. The time required for culture results often exceeds the time to clinical cure with empiric treatment, therefore, in almost all cases of UTI empirical antimicrobial treatment is initiated before the laboratory results of urine culture are available, contributing to increasing antimicrobial resistance due to misuse of antimicrobials (Wilson et al., 2004; Newell et al., 2000). Increasing multidrug resistance in bacterial uropathogens is an important and evolving public health challenge.

The serious increase in the prevalence of extended-spectrum beta lactamases (ESBL's) worldwide creates a need for effective and easy to perform screening methods for detection(Prakash et al., 2013, Yazdi M et al., 2012; Naiemi et al., 2009). ESBL producing organisms are those that hydrolyze the oxyimino beta-lactams and monobactams, but have no effect on the cephamycins and carbapenems. Also, the ESBL producers often also have resistance determinants to other antibiotic groups, leaving an extremely limited range of effective agents (Mukherjee et al., 2013). Detection of ESBL producers from urine specimens is essential because of transfer of drug resistant organisms to other patients. (Aggarwal et al., 2009)

Clinicians have tended to ignore the clinical importance of UTIs despite their significant prevalence, cost, morbidity, and increasing management problems. The reason is largely our opinion that uncomplicated UTIs are common yet not a serious problem, easy to diagnose, and effortless to treat. Antibioticresistant organisms causing UTI include Methicillin-resistant Staphylococcus aureus (MRSA), Methicillin-resistant coagulase- negative staphylococci (MRCoNS), vancomycin-resistant enterococci (VRE) and multidrug resistant Gram negative organisms. Candida species are frequently found as a colonizing organism and account for few clinical cases of UTI (Neal et al., 2008).

Recent guidelines from the Infectious Diseases Society of America recommended that empiric antibiotic therapy for UTIs should be based on local resistance data, drug availability, and antibiotic intolerance/allergy history of treated patients.(Gupta et al., 2011; Hooton et al., 2010) For uncomplicated cystitis, nitrofurantoin or trimethoprimsulfamethoxazole (TMP-SMX, if local resistance $\leq 20 \%$ ) can be used empirically, while fluoroquinolones, ceftriaxone, aminoglycosides, and carbapenems are appropriate for pyelonephritis and complicated UTI. Emerging antibiotic resistance among Enterobacteriaceae has posed challenges in choosing empiric regimens, especially in infections due to multidrug-resistant (MDR) Enterobacteriaceae (Sanchez et al., 2012) in the past decade, emerging resistance among the Enterobacteriaceae due to ESBL has been reported worldwide. (Qi et al., 2010) Therefore, regularly updated surveillance of local microbial prevalence and resistance patterns are needed to guide the empiric therapy for UTIs. Therefore, the present study was designed to identify etiological agents of urinary tract infections, detect ESBL producing uropathogens and study their antibiotic resistance profile.

\section{Objectives}

To isolate and identify uropathogenic gram negative bacilli.

To determine the antibiotic resistance profile of the isolates

To detect ESBL gram negative isolates 
As a baseline study to formulate hospital antibiotic policy and empirical treatment.

\section{Materials and Methods}

Type of study: Prospective study

Duration of the study: March 2018 to June 2018

Place of the study: Department of Microbiology, Mahavir Institute of Medical Sciences

\section{Inclusion criteria}

Patients suggestive of symptoms of UTI fever, burning micturation, frequency or urgency of urination, suprapubic discomfort, gross hematuria

Fever with pyuria and/or imaging evidence of UTI (cystitis, pyelonephritis, etc.)

Patients with urinary catheters and suggestive of UTI

\section{Exclusion criteria}

Patients with sexually transmitted infections, cervicitis, and vulvovaginitis (can present with symptoms similar to cystitis)

Patients with history of antibiotic therapy for UTI before sending specimen for culture

Patients not willing to participate in the study

Around 306 patients with the inclusion criteria were screened in the present study. The details of patient including name, age, gender, ward (for admitted cases) and brief clinical history were noted. Midstream urine (MSU) sample was collected in sterile, wide mouth, leakproof container and transported immediately to Microbiology laboratory. Catherized urine samples were collected as per standard guidelines. Semi-quantitative culture of urine was done on Cysteine Lactose Electrolyte Deficient (CLED) media, blood agar, and MacConkey agar by standard loop method. The culture plates were incubated at $37^{\circ} \mathrm{C}$ for 18-24 h under aerobic conditions. Identification of bacterial growth was confirmed by standard microbiological techniques (Forbes et al., 2007; Collee et al., 2008). Bacterial colony count more than $10^{5}$ colony-forming units $(\mathrm{CFU}) / \mathrm{ml}$ was taken as significant bacteriuria.

Antibiotic sensitivity testing was done by Kirby-Bauer disc diffusion method on Mueller-Hinton agar, as per Clinical and Laboratory Standards Institute guidelines. (Bauer et al., 1966; Clinical and Laboratory Standards Institute 2017) Antibiotic discs were procured from HiMedia, Mumbai, India. ESBLscreening and phenotypic confirmation was done by testing the strain against ceftazidime and ceftazidime/clavulanic acid, cefotaxime and cefotaxime/clavulanic acid). A difference of $>5 \mathrm{~mm}$ diameter of the zone of inhibition for combination disc in comparison to the ceftazidime/cefotaxime alone was considered as indicative of ESBL production. Escherichia coli ATCC 25922 for ESBL negative and Klebsiella pneumoniae 700603 for ESBL positive was used as reference strains. (Clinical and Laboratory Standards Institute 2017)

\section{Results and Discussion}

A total of 306 urine samples were collected from 82 male and 224 female patients. Significant bacteriuria was observed in $92.1 \%$ (282/306) samples. Out of the culture positive 282 specimens, female patients reported $72.4 \%$ growth whereas male patients reported $27.6 \%$ growth. Gender wise distribution of specimens and culture positivity is mentioned in Table 1. Out of 282 isolates, $96.1 \%$ isolates 
were Gram-negative, E. coli being predominant isolate whereas $3.9 \%$ were Gram-positive isolates (including Candida spp) as mentioned in Table 2.

The isolates showed least resistance to Imipenem (12\%), followed by Nitrofurantoin (24\%), Gentamicin (28\%) Piperacillin Tazobactam (36\%). Higher resistance was reported for Norfloxacin (80\%), Cefazolin (76\%), and Cotrimoxazole (68\%) Ciprofloxcin (64\%), Tetracycline (60\%). The details of antimicrobial sensitivity are mentioned in Table 3. Among the Gram negative isolates, $40.2 \%(109 / 271)$ were found to be ESBL producers (Table 4 ).

A total of 282 urine cultures were reported positive, 204 (72.4\%) females and 78 (27.6\%) male patients. Various research studies also conclude that UTI are more common in females as compared to males (Daniele et al., 2011, Dash et al., 2013).

Most of the uropathogenic bacteria are from the host's own gut flora and enter the bladder via the urethra. Shorter urethra in females as compared to males, with its proximity to anus, facilitates the bacteria to ascend in the urinary tract. (Yamamoto et 1997, Mitsumori et al., 1997) Also, sexually active women have an increased risk of UTI. About $20 \%$ of young women with a first UTI will have a recurrent infection. (Scholes et al., 2000)

Of the total 282 uropathogen isolates, Escherichia coli was the leading isolate with $70.2 \%(198 / 282)$ specimens reporting the growth, followed by Klebsiella pneumonia $19.8 \%$ (56/282), P. aeruginosa $4.25 \%$ (12/282), Citrobacter spp 1.06\%(03/282), Proteus spp $0.7 \%$ (02/282). Among gram positive uropathogens, majority were Coagulase negative Staphylococcus, followed by Staphylococcus aureus, Enterococcus and Candida spp.
Uropathogenic Escherichia coli (UPEC) from the gut are the cause of $80-85 \%$ of community-acquired urinary tract infections (Etienne et al., 2014; Schito et al., 2009). In uncomplicated UTIs, E.coli is the leading organism, whereas in complicated UTIs the bacterial spectrum is much broader including Gram-negative and Gram-positive and often multidrug resistant organisms.

Research studies suggest that $\mathrm{P}$ fimbriae contribute as virulence factors of $E$. coli strains to cause UTI, especially the more clinically severe forms. As per various researches, leading organisms involved in uncomplicated UTIs, after UPEC are Klebsiella pneumoniae, Staphylococcus saprophyticus, Enterococcus faecalis, group B Streptococcus, Proteus mirabilis, Pseudomonas aeruginosa, Staphylococcus aureus and Candida spp (Foxman et al., 2014, Nielubowicz et al., 2010, and Kline et al., 2011).

In the present study, gram negative isolates showed least resistance to Imipenem (12\%), followed by Nitrofurantoin (24\%), Gentamicin (28\%) Piperacillin Tazobactam (36\%).

A significant amount of resistance was documented to antibiotics like Norfloxacin (80\%), Cefazolin (76\%), and Cotrimoxazole (68\%) Ciprofloxcin (64\%), Tetracycline $(60 \%)$. In the current study, overall $40.2 \%$ (109/271) gram negative isolates were detected positive for ESBL production. E. coli was the predominant $77.06 \%$ (84/109) ESBL producing isolate. ESBL production has been reported ranging from $38.9 \%$ (Rishabh et al., 2018), 39.5\% (Vasumathi et al., 2016), 40\% (Babypadmini et al., 2004), 42\% (Babek et al., 2012), 44.5\% (Saeide et al., 2014) which is similar to our findings. However, even higher incidence of $58 \%$ and $84.6 \%$ has been reported by Mathur et al., (2002) \& Rejitha et al., (2014) respectively (Table 5). 
Table.1 Gender wise and department wise distribution of urine specimens

\begin{tabular}{|c|c|c|}
\hline & No. of specimens & Culture positive $(\mathrm{n}=282)$ \\
\hline Male & 82 & $78(27.6 \%)$ \\
\hline Female & 224 & $204(72.4 \%)$ \\
\hline Total & 306 & 282 \\
\hline \multicolumn{3}{|c|}{ OPD and IPD distribution of urine specimens } \\
\hline & No. of specimens & Culture positive $(\mathrm{n}=282)$ \\
\hline OPD & 232 & $196(69.5 \%)$ \\
\hline IPD & 74 & $86(30.5 \%)$ \\
\hline Total & 306 & 282 \\
\hline
\end{tabular}

Table.2 Distribution of uropathogens (Total isolates $n=282$ )

\begin{tabular}{|c|c|c|c|}
\hline S. no & Organism isolated & No. of isolates & ESBL positive** \\
\hline \multicolumn{4}{|c|}{ Gram negative $(n=271)$} \\
\hline 1. & Escherichia coli & 198 & 84 \\
\hline 2. & Klebsiella spp & 56 & 22 \\
\hline 3. & Pseudomonas aeruginosa & 12 & 02 \\
\hline 4. & Citrobacter spp & 03 & 01 \\
\hline 5. & Proteus spp & 02 & ----- \\
\hline \multicolumn{2}{|c|}{ Total isolates } & 271 & 109 \\
\hline \multicolumn{4}{|c|}{ Gram positive uropathogenic isolates $(\mathrm{n}=11)$} \\
\hline 1. & Staphylococcus aureus & 03 & \\
\hline 2. & Coagulase negative Staphylococcus (CoNS) & 05 & \\
\hline 3. & Candida spp & \multirow{2}{*}{02} & \\
\hline 4. & Enterococcus & & \\
\hline \multicolumn{2}{|r|}{ Total urinary isolates } & & 282 \\
\hline
\end{tabular}

** ESBL not tested in Gram positive isolates

Table.3 Antimicrobial susceptibility pattern of gram negative urinary pathogens $(n=271)$

\begin{tabular}{|c|c|c|c|}
\hline S. no & Antimicrobial & Sensitive (\%) & Resistant (\%) \\
\hline 1. & Norfloxacin $10 \mu \mathrm{g}$ & $20 \%$ & $80 \%$ \\
\hline 2. & Cefazolin $30 \mu \mathrm{g}$ & $24 \%$ & $76 \%$ \\
\hline 3. & Cotrimaxazole $25 \mu \mathrm{g}$ & $32 \%$ & $68 \%$ \\
\hline 4. & Ciproflaxacin $5 \mu \mathrm{g}$ & $36 \%$ & $64 \%$ \\
\hline 5. & Tetracycline 30ug & $40 \%$ & $60 \%$ \\
\hline 6. & Ceftriaxone $30 \mu \mathrm{g}$ & $54 \%$ & $46 \%$ \\
\hline 7. & Ceftazidime $30 \mu \mathrm{g}$ & $58 \%$ & $42 \%$ \\
\hline 8. & Piperacillin-Tazobactam 100/10 $\mu \mathrm{g}$ & $64 \%$ & $36 \%$ \\
\hline 9. & Gentamicin $10 \mu \mathrm{g}$ & $72 \%$ & $28 \%$ \\
\hline 10. & Nitrofurantoin $300 \mu \mathrm{g}$ & $76 \%$ & $24 \%$ \\
\hline 11. & Imipenem $10 \mu \mathrm{g}$ & $88 \%$ & $12 \%$ \\
\hline
\end{tabular}


Table.4 Antimicrobial Sensitivity of ESBL isolates ( $\mathrm{n}=109)$

\begin{tabular}{|l|}
\hline S. no \\
\hline 1. \\
\hline 2. \\
\hline 3. \\
\hline
\end{tabular}

\section{Antimicrobial}

Imipenem $10 \mu \mathrm{g}$

Nitrofurantoin $300 \mu \mathrm{g}$

Piperacillin-Tazobactam 100/10 $\mu \mathrm{g}$

\section{Sensitive ESBL isolates (\%)}

$90(82.5)$

$85(77.9 \%)$

$78(71.5 \%)$

Table.5 Comparison of community vs hospital ESBL isolates

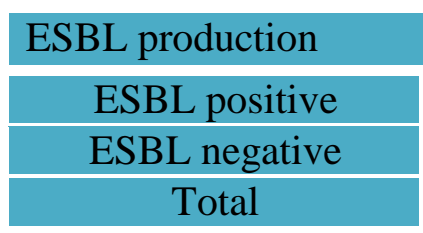

\section{Community acquired UTI}

31
165
196

196

\section{Hospital acquired UTI Total}

\begin{tabular}{|c|c|}
\hline 78 & 109 \\
\hline 08 & 173 \\
\hline 86 & 282 \\
\hline
\end{tabular}

The $\mathrm{p}$ value is $<0.00001$.The result is significant at $\mathrm{p}<0.05$ by Chi square test

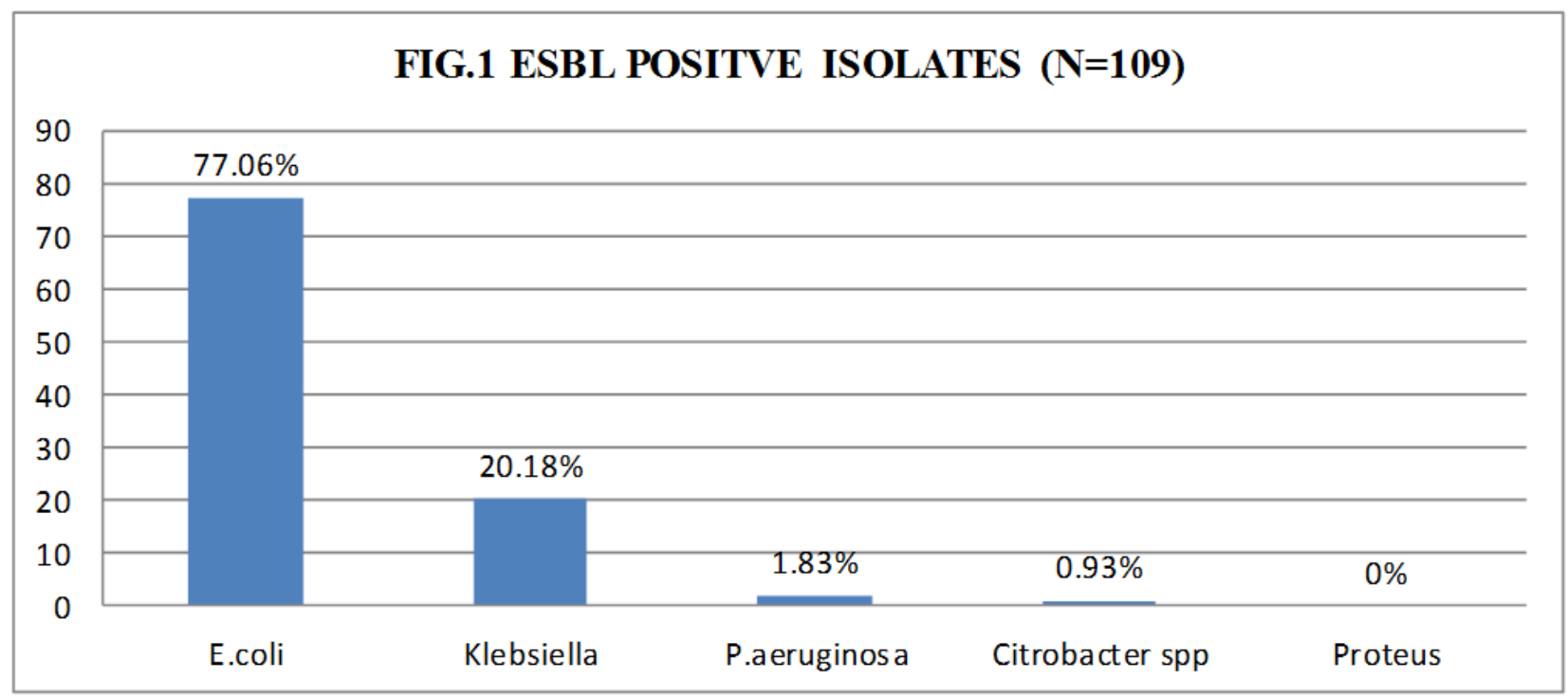

FIG.2 Sensitivity pattem of ESBL isolates (\%)

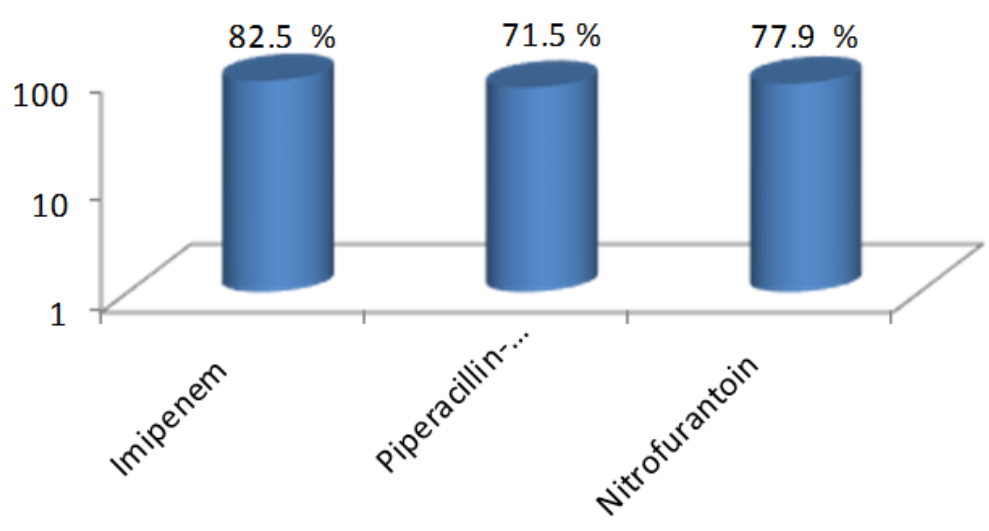

- Sensitive is olates (\%) 
The local epidemiology, study population, patterns and protocols of antibiotic usage have a remarkable influence on differences in the ESBL pattern documented in the above mentioned studies.

In the present study, hospital ESBL isolates i.e $71.5 \%(78 / 109)$ were significantly higher than community ESBL isolates $28.5 \%$ (31/109). Higher incidences of ESBL uropathogens in hospitalized patients as compared to community settings are reported by Babypadmini et al., (2004) and Babek et al., (2012). Factors predisposing to emergence and transmission of ESBL organisms include the duration of exposure to broad spectrum antibiotics, length of stay in hospital, severity of underlying illness, use of invasive devices such as urinary catheters, or surgery. (Flaherty et al., 1996) It is well known that ESBL producing bacteria cause infections in hospitalized patients but nowdays ESBL isolates are reported from community infections also (Nesher et al., 2007). Mahesh et al., (2010) and Taneja et al., (2008) reported higher ESBL isolates around $56.2 \%$ and $36.5 \%$ from community setting as compared to our study.

ESBL producing organisms causing community UTI can lead to treatment failure or delayed clinical response. Furthermore, these isolates being multidrug resistant, antimicrobials like amino glycosides, quinolones and cotrimoxazole are often ineffective clinically (Auer et al., 2010) Carbapenems are generally considered the drug of choice for the treatment of ESBL infections (Pitout et al., 2008; Rodriguez et al., 2012). Therefore, on basis of above considerations, in the present study we have analyzed sensitivity of ESBL producers for imipenem, nitrofurantion and piperacillin tazobactam which can retain their activity even against ESBL pathogens. In the present study $82.5 \% \quad(90 / 109)$ ESBL producing isolates were sensitive to Imipenem. Also, as per the results in the current study, nitrofurantoin can also be considered as a good treatment option, the resistance being less documented. The findings are in consensus with studies done by Bajpai et al., (2014), Sasirekha et al., (2013), and Khameneh et al., (2009) which have reported nitrofurantion as a suitable agent for the first line treatment of the community acquired UTI.

Piperacillin-tazobactam (PTZ) is one of the most frequently utilized agents for empiric gram negative bacterial coverage and retains activity against ESBL producers.

Furthermore, antimicrobial stewardship practices encourage the use of carbapenem sparing treatment regimens for infections due to ESBL gram negative infections. Results from this study suggest that PTZ is effective in the treatment of urinary tract infection caused by ESBL when the in vitro test indicates susceptibility. Randomized controlled trial by Seo et al., (2017) also confirms effectiveness of PTZ in ESBL producing uroparthogenic E. coli.

ESBL uropathogens are isolated from community as well as hospital settings. ESBL producers generally do not respond to the usually prescribed empirical therapy. Presently, alternative antimicrobial therapy to treat ESBL positive UTI on outpatient basis is limited. Imipenem was the most effective drug; however, it should not be administered as empirical drug unless infection is life threatening, as carbapenems are considered the drug of last resort. But alternatives for the treatment of ESBL-producing bacteria are urgently needed to suppress the emergence of carbapenem resistance. Results from this study suggest that Piperacillin-tazobactam, nitrofurantoin, are also effective in the treatment of UTI caused by ESBL pathogens. 


\section{References}

Aggarwal R, Chaudhary U, Sikka R. Detection of extended spectrum Blactamase production among uropathogens. J Lab Physicians 2009; 1:7-1014.

Amdekar S, Singh V, Singh DD. Probiotic therapy: immunomodulating approach toward urinary tract infection. Current microbiology 2011; 63 (5): 484-90.

Auer S, Wojna A, Hell M. Oral Treatment Options for Ambulatory Patients with Urinary Tract Infections Caused by Extended-Spectrum beta-Lactamase Producing Escherichia coli. Antimicro Agents Chemo 2010, 54:4006-8.

Babak Pourakbari, Farzad Ferdosian, Shima Mahmoudi, Mostafa Teymuri, Farah Sabouni, Hossein Heydari et al., Increase resistant rates and ESBL production between $E$. coli isolates causing Urinary Tract Infection in young patients from Iran. Brazilian Journal of Microbiology, 2012: 766769

Babypadmini S, Appalaraju B. Extended spectrum $\beta$ lactamases in urinary isolates of Escherichia coli and Klebsiella pneumoniae Prevalence and susceptibility pattern in a tertiary care hospital. Indian J Med Microbiol. 2004; 22:172-4.

Bagshaw SM, Laupland, KB. Epidemiology of intensive care unit-acquired urinary tract infections. Current Opinion in Infectious Diseases 2006; 19 (1): 67-71.

Bajpai, T., Pandey, M., Varma, M. and Bhatambare, G. S. Prevalence of extended spectrum beta-lactamase producing uropathogens and their antibiotic resistance profile in patients visiting a tertiary care hospital in central India: Implications on empiric therapy. Indian J. Pathol. Microbiol 2014.57, 407-12.
Bauer AW, Kirby WMM, Sherris JC, Turck M. Antibiotic susceptibility testing by a standardized single disc method. Am. J. Clin. Pathol. 1966; 45:493-496

Clinical and Laboratory Standards Institute. Performance Standards for Antimicrobial Susceptibility testing; CLSI document M100-S23.Wayne, PA: Clinical and Laboratory Standards Institute; 2017.

Collee JG, Miles RS, Watt B. Mackie \& McCartney $\mathrm{s}$ Practical Medical Microbiology14th Ed. In: JG Collee, AG Fraser, BP Marmion, A Simmons, Editors. Churchill Livingstone: Indian Reprint; 2008

Daniele Minardi Gianluca d'Anzeo Daniele Cantoro Alessandro Conti Giovanni Muzzonigro. Urinary tract infections in women: etiology and treatment options. International Journal of General Medicine 2011; 4: 333-343

Dash M, Padhi S, Mohanty I, Panda P, Parida B. Antimicrobial resistance in pathogens causing urinary tract infections in a rural community of Odisha, India J Family Community Med 2013; 20(1): 20-6.

Etienne M, Lefebvre E, Frebourg N, Hamel H, Pestel-Caron M, Caron F. Antibiotic treatment of acute uncomplicated cystitis based on rapid urine test and local epidemiology: lessons from a primary care series. BMC Infect Dis. 2014; 14:1-8.

Flaherty JP, Weinstein RA. Nosocomial infection caused by antibiotic-resistant organisms in the intensive-care unit. Infect Control Hosp Epidemiol. 1996; 17:236-248.

Flores-Mireles AL; Walker JN; Caparon M; Hultgren, SJ. Urinary tract infections: epidemiology, mechanisms of infection and treatment options. Nature Reviews. Microbiology 2015; 13(5): 269-84. 
Forbes BA, Sahm DF, Weissfield AS. Bailey and Scotts Diagnostic Microbiology. $12^{\text {th }}$ ed. USA: Mosby Elsevier; 2007.

Foxman B. Urinary tract infection syndromes: occurrence, recurrence, bacteriology, risk factors, and disease burden. Infect Dis Clin North Am. 2014; 28:1-13.

Gupta, K., T. M. Hooton, K. G. Naber et al., International clinical practice guidelines for the treatment of acute uncomplicated cystitis and pyelonephritis in women: a 2010 update by the infectious diseases society of America and the European society for microbiology and infectious diseases, Clinical Infectious Diseases 2011; 52(5): 103-120

Hooton, T. M., S. F. Bradley, D. D. Cardenas et al., Diagnosis, prevention, and treatment of catheter-associated urinary tract infection in adults: 2009 international clinical practice guidelines from the infectious diseases society of America, Clinical Infectious Diseases 2010; 50(5): 625-663

Khameneh, Z R. and Afshar, A. T. Antimicrobial susceptibility pattern of urinary tract pathogens. Saudi J. kidney Dis. Transplant 2009; 20: 251-3.

Kline KA, Schwartz DJ, Lewis WG, Hultgren SJ, Lewis AL. Immune activation and suppression by group B Streptococcus in a murine model of urinary tract infection. Infect Immun. 2011; 79:3588-3595.

Mahesh E, Ramesh D, Indumathi VA, et al., Risk Factors for Community Acquired Urinary Tract Infection caused by ESBL-producing Bacteria. JIACM 2010; 11:271-6.

Mathur P, Kapil A, Das B, Dhawan B. Prevalence of extended spectrum $\beta$ lactamase producing gram negative bacteria in a tertiary care hospital. Indian $\quad J \quad$ Med Res. 2002; 115:153-7.
Mitsumori K, Terai A, Yamamoto S, Yoshida O. Virulence characteristics and DNA fingerprints of Escherichia coli isolated from women with acute uncomplicated pyelonephritis. J Urol. 1997; 158(6):2329-2332

Mukherjee M, Basu S, Mukherjee SK, Majumder M. Multidrug-resistance and extended spectrum beta-lactamase production in uropathogenic E. coli which were isolated from hospitalized patients in Kolkata, India. J Clin Diagn Res 2013; 7:449-53.

Naiemi NA, Murk JL, Savelkoul PH, Vandenbroucke-Grauls CM, Debets Ossenkopp YJ. Extended-spectrum beta-lactamases screening agar with AmpC inhibition. Eur J Clin Microbiol Infect Dis 2009; 28:989-90

Neal DE Jr. Complicated urinary tract infections. Urol Clin North Am 2008; 35: $13-22$

Nesher L, Novack V, Riesenberg F, et al., Regional community-acquired urinary tracts in Israel; diagnosis, pathogens, and antibiotics guidelines adherence: A prospective study. International J Infect Dis 2007; 11:245-50.

Newell A, P. Riley, and M. Rodgers, Resistance patterns of urinary tract infections diagnosed in a genitourinary medicine clinic. International Journal of STD and AIDS 2000; 11(8): 499-500.

Nicolle LE. Uncomplicated urinary tract infection in adults including uncomplicated pyelonephritis. Urol Clin North Am 2008; 35 (1): 1-12

Nielubowicz GR, Mobley HL. Host-pathogen interactions in urinary tract infection. Nature Rev Urol. 2010; 7:430-441.

Pitout JDD, Laupland KB. Extendedspectrum $\quad \beta$-lactamase-producing Enterobacteriaceae: an emerging publichealth concern. Lancet Infect Dis. 2008; 8:159-166. 
Prakash D, Saxena RS. Distribution and Antimicrobial Susceptibility Pattern of Bacterial Pathogens Causing Urinary Tract Infection in Urban Community of Meerut City, India. ISRN Microbiology. 2013; 2013:749629.

Qi, C., V. Pilla, J. H. Yu, and K. Reed.Changing prevalence of Escherichia coli with CTX-M-type extended-spectrum $\beta$-lactamases in outpatient urinary E. coli between 2003 and 2008.Diagnostic Microbiology and Infectious Disease 2010; 67(1):87-91.

Rejitha IM, G Sucilathangam, G Velvizhi. Urinary Tract Infection (UTI) In the Elderly - A Clinical and Microbiological Study. Indian Journal of Applied Research Medical Science, April 2014; 4(4): 465-467.

Rishabh Rajput and Surendra Sarsaiya. Significance of Regional Antibiogram and MDR of ESBL Producing Uropathogens Infecting Nonhospitalized Patients: Gurugram. Int.J.Curr.Microbiol.App.Sci 2018; 7(2): 1114-1126

Rodriguez-Bano J, Navarro MD, Retamar P, et al., $\quad \beta$-lactam $/ \beta$-lactam inhibitor combinations for the treatment of bacteremia due to extended-spectrum $\beta$ -lactamase-producing Escherichia coli: a post hoc analysis of prospective cohorts. Clin Infect Dis. 2012; 54(2):167-174.

Saeide Saeidi, Mehdi Ghamgosha, Ramezan Ali Taheri, Yasub Shiri, Mahmood Solouki, Kazem Hassanpour, Gholamreza Farnoosh. Phenotypic and genotypic detection of extendedspectrum $\quad \beta$-lactamase $\quad$ (ESBL) producing Escherichia coli isolated from urinary tract infections in Zabol, Iran. Journal of Coastal Life Medicine 2014; 2(9): 732-737

Salvatore S, Cattoni E, Siesto G, Serati M, Sorice P, Torella M. Urinary tract infections in women. European journal of obstetrics, gynecology, and reproductive biology 2011;156(2): $131-6$

Sanchez, G. V., R. N. Master, J. A. Karlowsky, and J. M. Bordon.In vitro antimicrobial resistance of urinary Escherichia coli isolates among U.S. outpatients from 2000 to 2010.Antimicrobial Agents and Chemotherapy 2012; 56(4): 21812183.

Sasirekha, B. Prevalence of ESBL, AmpC $\beta$ lactamases and MRSA among uropathogens and its antibiogram. EXCLI J 2013; 12:81-8

Schito GC, Naber KG, Botto H, Palou J, Mazzei T, Gualco L, et al., The ARESC study: an international survey on the antimicrobial resistance of pathogens involved in uncomplicated urinary tract infections. Int $\mathbf{J}$ Antimicrob Agents. 2009; 34:407-13.

Scholes D, Hooton TM, Roberts PL, Stapleton AE, Gupta K, Stamm WE. Risk factors for recurrent urinary tract infection in young women. J Infect Dis. 2000; 182(4):1177-1182.

Seo YB, Lee J, Kim YK, Lee SS, Lee JA, Kim HY, Uh Y, Kim HS, Song W. Randomized controlled trial of piperacillin-tazobactam, cefepime and ertapenem for the treatment of urinary tract infection caused by extendedspectrum beta-lactamase-producing Escherichia coli. BMC Infect Dis. 2017 Jun 7; 17(1):404

Sievert DM, Ricks P, Edwards JR, et al., Antimicrobial-resistant pathogens associated with healthcare-associated infections: summary of data reported to the National Healthcare Safety Network at the Centers for Disease Control and Prevention, 2009-2010. Infect Control Hosp Epidemiol 2013; 34 (1): 1-14. 
Taneja N, Rao P, Arora J, et al., Occurrence of ESBL \& Amp-C $\beta$-lactamases \& susceptibility to newer antimicrobial agents in complicated UTI. Indian J Med Res 2008; 127: 85-8.

Vasumathi A, Thenmozhi Valli PR, Senthamarai S. Extended Spectrum B Lactamase (ESBL) Producing Uropathogens in the Intensive Care Unit in A Tertiary Care Hospital, Tamilnadu IOSR-JDMS 2016;15 (2): 66-68

Wilson, M. L., and L. Gaido. Laboratory diagnosis of urinary tract infections in adult patients," Clinical Infectious Diseases 2004; 38(8): 1150-1158.

Yamamoto S, Tsukamoto T, Terai A, Kurazono H, Takeda Y, Yoshida O. Genetic evidence supporting the fecalperineal-urethral hypothesis in cystitis caused by Escherichia coli. J Urol. 1997; 157(3):1127-1129.

Yazdi M, Nazemi A, Mirinargasi M, Jafarpour M, Sharifi SH. Genotypic versus Phenotypic methods to detect extended-spectrum betalactamases (ESBLs) in uropathogenic Escherichia coli. Ann Biol Res 2012; 3:2454-8.

\section{How to cite this article:}

Gitanjali Kailas Badave, Qader Ahmed Jalily and Sasikala, G. 2018. A Study of Uropathogenic ESBL Producing Gram Negative Bacilli in a Teaching Hospital. Int.J.Curr.Microbiol.App.Sci. 7(09): 919-929. doi: https://doi.org/10.20546/ijcmas.2018.709.111 